\title{
The fate of aspergilloma patients after surgical treatment- experience from 22 cases
}

\author{
Katriina Pihlajamaa ${ }^{1}$, Veli-Jukka Anttila ${ }^{2}$, Jari V. Räsänen ${ }^{3,4}$, Juha T. Kauppi ${ }^{3,4}$, Ulla Hodgson ${ }^{1}$ \\ ${ }^{1}$ Heart and Lung Center, ${ }^{2}$ Inflammation Center, Helsinki University Hospital, Helsinki University, Helsinki, Finland; ${ }^{3}$ Department of General \\ Thoracic and Esophageal Surgery, Helsinki University Hospital, Helsinki, Finland; ${ }^{4}$ Department of Surgery, Clinicum, University of Helsinki, \\ Helsinki, Finland \\ Contributions: (I) Conception and design: VJ Anttila, U Hodgson, K Pihlajamaa; (II) Administrative support: JV Räsänen, U Hodgson; (III) Provision \\ of study materials or patients: JV Räsänen, JT Kauppi, U Hodgson; (IV) Collection and assembly of data: K Pihlajamaa; (V) Data analysis and \\ interpretation: K Pihlajamaa, VJ Anttila, U Hodgson; (VI) Manuscript writing: All authors; (VII) Final approval of manuscript: All authors. \\ Correspondence to: Katriina Pihlajamaa. HUS Meilahti Triangle Hospital, PO Box 372, 00029 HUS, Helsinki, Finland. Email: katriina.pihlajamaa@helsinki.fi.
}

Background: Patients with pulmonary aspergillomas occasionally undergo surgery but it is somewhat unclear who of these patients benefit from surgical treatment.

Methods: We retrospectively evaluated all 22 patients that underwent surgery in Helsinki University Central Hospital between 2004 and 2017. We assessed their clinical backgrounds, anti-fungal medication, indication for surgery, complications, recurrent infections and survival.

Results: Of the 22 patients, 14 male and 8 female, mean age 56, an underlying pulmonary disease was present in 20. On immunosuppressive medication were 8 (36\%). Most received anti-fungal medication preoperatively $(n=12)$ and/or postoperatively $(n=17), 3$ patients did not receive anti-fungal medication. Length of the medication periods were diverse. Main indication for surgery was haemoptysis. One inhospital-death occurred, and other complications included prolonged air-leak, postoperative pneumonia, pneumothorax and pneumomediastinum. No Aspergillus empyema or pleurites occurred. Five-year survival was 54\%. One in-hospital-death and one other death were the result of Aspergillus disease, other deaths were unrelated to Aspergillus. Recurrent disease occurred in four cases. Three of these patients were asthma patients with allergic bronchopulmonary aspergillosis (ABPA).

Conclusions: Overall results of surgery in this cohort were good and number of complications was low. Therapy with antifungals was diverse. Surgical treatment of aspergilloma can be life-saving for patients suffering of haemoptysis, and patients with restricted disease and well-preserved pulmonary capacity may benefit from surgery. Careful patient selection is crucial.

Keywords: Pulmonary aspergillosis; thoracic surgery; haemoptysis; antifungal agents

Submitted May 22, 2019. Accepted for publication Aug 27, 2019.

doi: $10.21037 /$ jtd.2019.09.48

View this article at: http://dx.doi.org/10.21037/jtd.2019.09.48

\section{Introduction}

Chronic pulmonary aspergillosis is a rather uncommon disease often affecting patients with prior or current pulmonary disease. These patients are usually nonimmunocompromised or mildly immunocompromised (1). The morphological appearance of a fungal ball which typically forms in a pre-existing pulmonary cavity due to saprophytic colonization of Aspergillus consists almost entirely of fungal hyphae and extracellular matrix and is called pulmonary aspergilloma. Several forms of chronic pulmonary aspergillosis can occur with an aspergilloma. Belcher and Plummer in 1960 classified aspergillomas as simple or complex, in which simple aspergilloma is a single fungal ball in a single pulmonary cavity that does not progress over months and causes few or no symptoms; serological evidence of Aspergillus may be absent $(2,3)$. 
Table 1 Patient characteristics

\begin{tabular}{|c|c|}
\hline Characteristic & Number \\
\hline Total number of patients & 22 \\
\hline Age, median & 56 [37-75] \\
\hline \multicolumn{2}{|l|}{ Gender (n) } \\
\hline Male & 14 \\
\hline Female & 8 \\
\hline \multicolumn{2}{|l|}{ Pulmonary disease (n) } \\
\hline Asthma & 7 \\
\hline COPD & 5 \\
\hline Previous tuberculosis & 5 \\
\hline Other previous infection & 2 \\
\hline Pneumothorax & 2 \\
\hline Emphysema & 1 \\
\hline Previous aspergilloma & 1 \\
\hline \multicolumn{2}{|l|}{ Comorbidities (n) } \\
\hline Rheumatological disease & 6 \\
\hline Malignancy (current or previous) & 5 \\
\hline Diabetes mellitus & 2 \\
\hline Chronic neutropenia & 2 \\
\hline Stem cell transplantation & 2 \\
\hline Solid organ transplant & 1 \\
\hline Alcohol abuse & 1 \\
\hline
\end{tabular}

Contemporary terminology for complex aspergilloma is chronic cavitary pulmonary aspergillosis (CCPA). CCPA is associated with high morbidity and mortality $(1,4,5)$ and results of surgical resection of an aspergilloma in CCPA, compared with those for simple aspergilloma, are considerably worse $(2,6-8)$.

When a pulmonary cavity with an aspergilloma exists, this can result in repetitive haemosputum or lifethreatening massive haemoptysis (4,9-11) and results of antifungal treatment are usually disappointing (12). Spontaneous lysis of an aspergilloma happens occasionally, and occurs in $5 \%$ to $10 \%$ of cases $(13,14)$. Options for treating haemoptysis are either radiological embolization of artery or surgical resection; the latter, however, is the only option for curative treatment. The surgical procedure can often be challenging due to ongoing inflammatory process resulting in formation of intrathoracic adhesions, and the risk for postoperative complications remains high $(2,15)$. Selecting the right patients for surgical treatment is thus crucial. Despite several decades' experience, the indications remain somewhat unclear as does optimal use of antifungal treatment combined with surgery.

Here, we evaluated the background and the clinical and surgical long- and short-term outcome of patients who underwent surgery for pulmonary aspergillosis in Helsinki University Central Hospital between 2004 and 2017. The aim was to analyze early and long-term results of surgical treatment for pulmonary aspergilloma.

\section{Methods}

\section{Study population}

From 2004 to 2017, 22 patients underwent surgery for pulmonary aspergilloma in Helsinki University Hospital and we retrospectively assessed their clinical and surgical characteristics. Patient identification in the hospital registry utilized ICD-codes B44.0 to B44.9. All the data came from hospital database and medical records. We examined their clinical backgrounds: age, gender, underlying pulmonary disease, immunosuppressive medication or other cause of immunosuppression, Aspergillus-related laboratory findings, pulmonary function test results, anti-fungal medication (if any) and its duration pre- and postoperatively, and indication for surgery. We assessed peri- and postoperative results: operative procedure, duration of surgery, intraoperative blood loss, use of omentum or latissimus dorsi to fill the remaining empty space during surgery, perioperative complications, duration of hospital stay, postoperative complications, recovery from surgery, recurrent Aspergillus infection, survival, and reason of death if not alive in October 2018.

The study protocol was approved by the research board of the Heart and Lung Centre of Helsinki University Hospital.

\section{Results}

\section{Clinical characteristics}

Of the 22 patients 14 were male, 8 female, mean age 56 [37-75]. An underlying pulmonary disease was evident in 20 , but 2 patients showed no respiratory disease. Underlying pulmonary diseases and comorbidities are listed in Table 1. The most common pulmonary conditions were asthma (7 patients) and COPD (5 patients). A pre-existing 

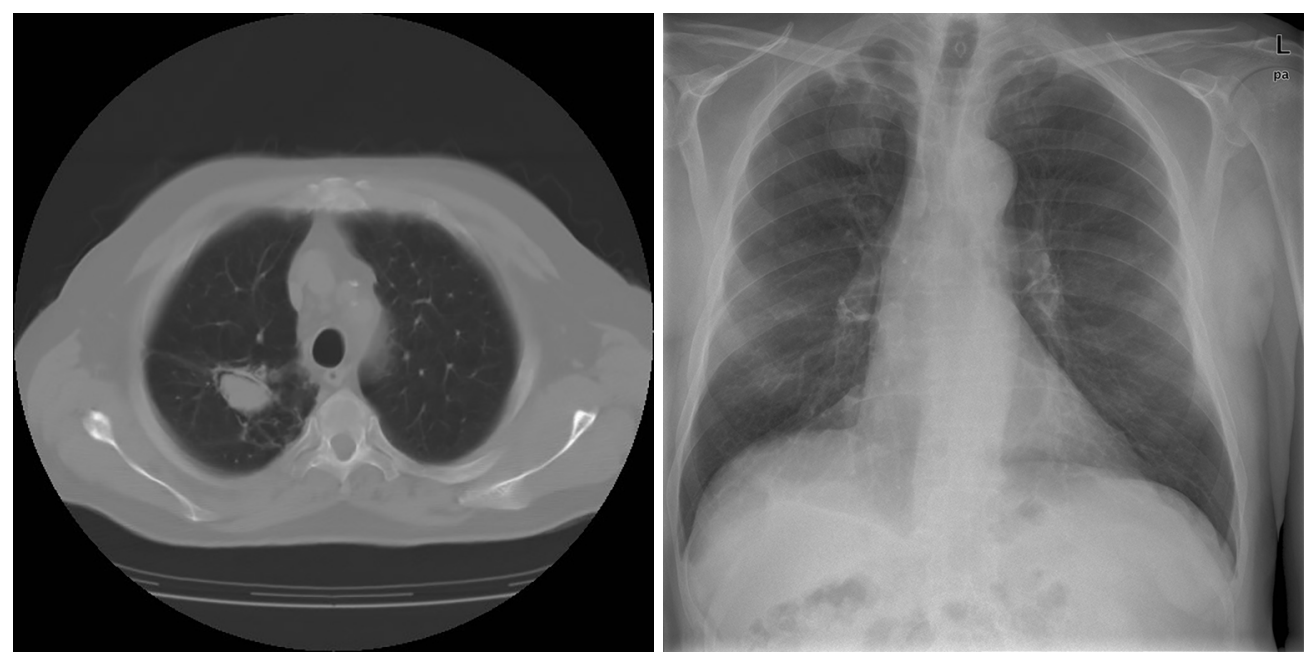

Figure 1 A 62-year-old male with history of pulmonary tuberculosis. Simple aspergilloma. No immunosuppression. Lobectomy of right upper lobe.

pulmonary cavity apparent in previous thoracic X-ray was evident in 11 patients. Other common co-morbidities included rheumatological diseases-rheumatoid arthritis or ankylosing spondylitis-and elevated blood pressure. Of the two patients with no any evident lung disease one had chronic idiopathic non-immunological neutropenia, collagenosis and immunosuppressive treatment; the other only elevated blood pressure as a diagnosed co-morbidity.

\section{Immunosuppression}

At diagnosis, eight (36\%) patients received immunosuppressive treatment (more than $5 \mathrm{mg}$ prednisolone daily). Four of them received medication for rheumatological disease, one due to liver transplantation, one had immunological neutropenia and high-dose steroid treatment, one received repeated steroid courses for asthma, and one had undergone an allogenic stem cell transplantation due to Hodgkin disease and was suffering graft-versus-host disease (GVHD). Diabetes mellitus type II was the diagnosis in two patients and one was known to abuse alcohol. Five patients had previous or current malignant disease. One of them underwent surgery for aspergilloma prior to chemotherapy for oesophageal carcinoma, one had Hodgkin disease and allogenic stem cell transplantation, one patient had been operated on breast cancer and one for renal carcinoma, and one patient had suffered mediastinal embryonal carcinoma and undergone autological stem cell transplantation. These malignancies were considered cured, except for the case of oesophageal carcinoma. Among the 8 patients receiving immunosuppressive medication 6 had single aspergilloma and no evidence of progressive disease. Two of these patients had more than one nodule at the time of diagnosis.

\section{Preoperative lung function}

Lung-function test results were available in all but one patient preoperatively. Average forced vital capacity (FVC) as percent of predicted was $85 \%$ (range, $30-113 \%$ ) and average forced expiratory volume in one second (FEV1) as percent of predicted was $78 \%$ (range, 20-114\%). Diffusion capacity results were available for 16 patients, with average diffusion capacity as percent of predicted being $74 \%$ (range, $52-112 \%)$.

\section{Diagnosis}

Radiological findings usually raised suspicion of an aspergilloma. Radiological images of three of our patients can be seen in Figures 1-3. Radiological findings were not quite typical in all cases, but Aspergillus was suspected to be the cause. In 6 cases Aspergillus-positive sputum culture together with radiological findings and in $4 / 6$ patients also elevated Aspergillus fumigatus IgG-levels was considered to be sufficient evidence of aspergilloma. If sputum samples were negative or unavailable, or radiology suggestive of another diagnosis, further examinations were carried out. Bronchoscopy with bronchoalveolar lavation was performed 

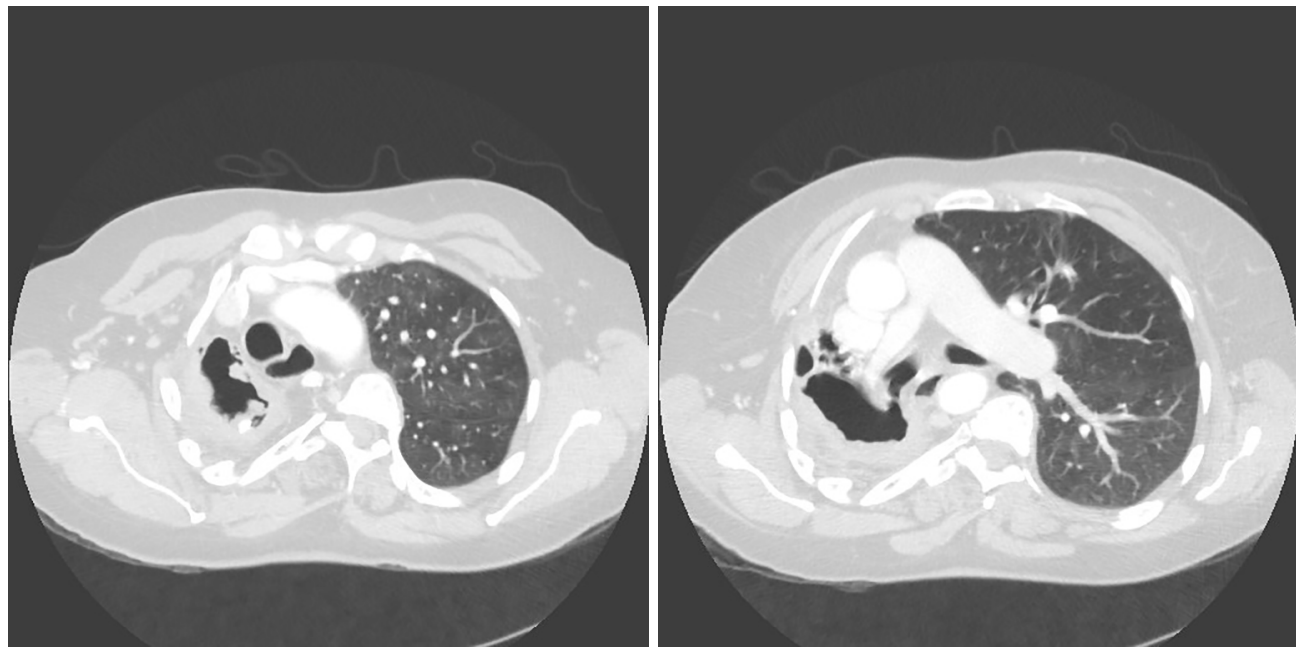

Figure 2 A 51-year-old female with history of pulmonary tuberculosis, grossly destroyed right lung. No immunosuppression. Hemoptysis. Pulmectomy of right lung.
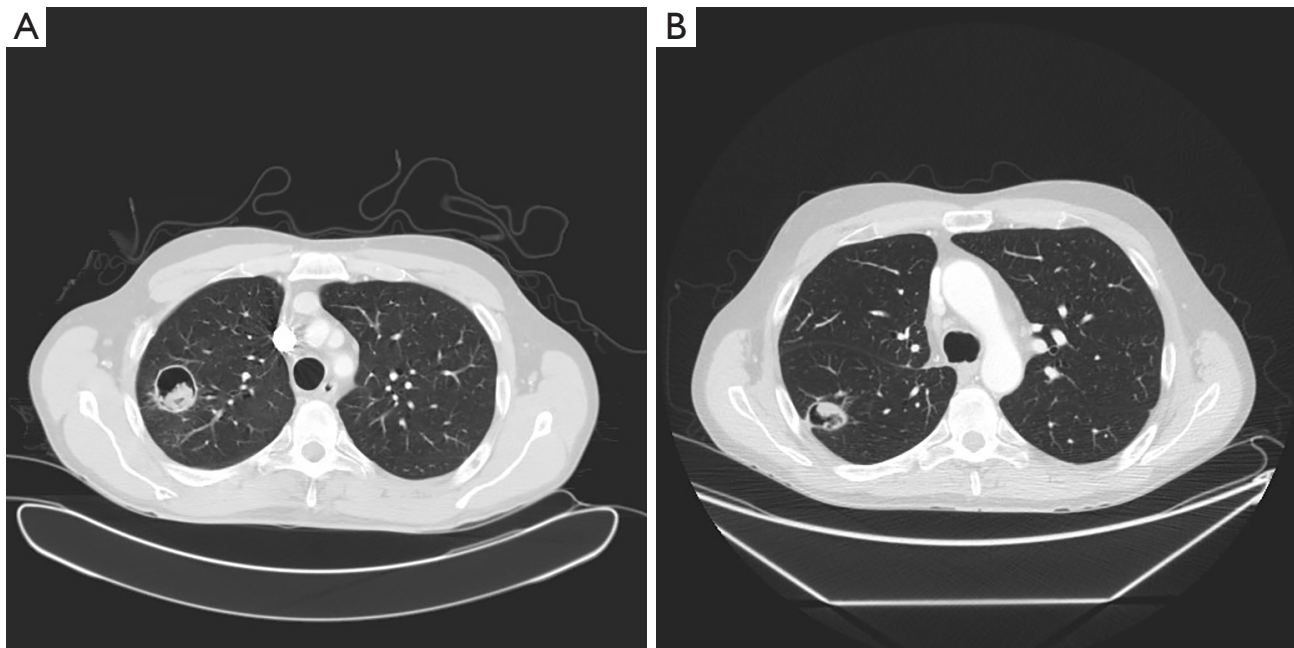

Figure 3 A 48-year-old male with history of asthma, no immunosuppression. Hemoptysis. (A) Before operation (lobectomy of right upper lobe); (B) recurrence in right lower lobe.

for 7 patients to obtain samples for culture, and they turned out to be positive for Aspergillus in 3/7. Samples for culture were obtained by CT-guided biopsy in 10 cases, however in three cases it did not confirm the diagnosis. Among the 22 patients, $16(73 \%)$ had a preoperative diagnosis of pulmonary aspergillosis by culture. Two of the patients having no positive cultures before surgery had elevated blood Aspergillus fumigatus IgG and IgE levels. Aspergillus fumigatus IgG levels in blood were elevated in 8 of our patients but had been analysed only in 10; Aspergillus fumigatus $\operatorname{IgE}$-levels had been analysed in 9 patients and proved to be elevated in 5 . In four cases, any microbiological or serological evidence of Aspergillus before surgery was absent. Despite missing verification of Aspergillus-disease these patients went through operation either because the suspicion of such disease was strong, or malignancy was considered to be possible diagnosis.

Histopathological tissue samples taken during surgery confirmed the diagnosis, with species growing in culture being Aspergillus fumigatus in all cases. Susceptibility testing 
Table 2 Surgical procedure

\begin{tabular}{lc}
\hline Operative characteristics & No. of patients \\
\hline Lobectomy only & 9 \\
With segmentectomy & 2 \\
Wedge resection only & 2 \\
With segmentectomy & 1 \\
Segmentectomy only & 6 \\
Pneumonectomy & 1 \\
Fenestration and resection & 1 \\
Operative time (minutes), median & $210[23-559]$ \\
Intraoperative blood loss (mL), median & $400[30-4,380]$ \\
Postoperative hospital stay (days), median & 12 [6-42] \\
\hline
\end{tabular}

results were available in eight (36\%) cases. No resistance against voriconazole was detectable, but susceptibility to posaconazole was lowered in two and to itraconazole in two cases.

Several of these aspergillomas treated surgically had formed in previously severely destroyed lung tissue, but with no evident Aspergillus in the surrounding lung parenchyma reported by the pathologist postoperatively; in most cases aspergillomas had remained stable for months prior to surgery. Thus, according to diagnostic criteria of CCPA as presented by Denning et al. $(3,16)$, the majority of our surgical patients did not have CCPA. In retrospective analysis CCPA-patients suffering from progressive disease accounted for $32 \%(7 / 22)$ of these surgical patients. One patient had two sites of a suspected aspergilloma preoperatively, in others only one site-but in some cases in largely destroyed lung parenchyma-was detected preoperatively.

\section{Operative indications and procedures}

Reasons for referring the patient for surgery included haemoptysis, recurrent infections, or an indefinite radiological finding. Indication for surgery was haemoptysis in $10(45 \%)$ patients. Other symptoms of infection or recurrent infections were the indication in eight (36\%) patients, two of whom had bronchopleural fistulae. In one patient an aspergilloma was evident in a CT scan while diagnosing oesophageal carcinoma, and he underwent surgery prior to chemotherapy to reduce the risk of fulminant fungal infection during immunosuppression.
Three patients were asymptomatic but had a thoracic X-ray for other reasons. In one case, the X-ray was very typical for simple aspergilloma but in the other two Aspergillus was considered a possible cause of an indefinite finding in a CT scan, but also preoperatively considered as possible diagnosis were an abscess caused by another infective agent or a malignancy.

The thoracic surgeon decided on surgical technique and extent. When only a small lesion of $3 \mathrm{~cm}$ or less existed, a wedge-resection or segmentectomy was the choice. Lobectomy occurred for any larger infiltration if lung capacity was sufficient, and in one case pulmonectomy was necessary because the lung was severely destroyed due to previous tuberculosis and scarring. If the remaining empty space needed filling, the surgeon decided whether to use latissimus dorsi flap or omentum during the operation. According to medical records, no intraoperative spillage of Aspergillus into the pleural cavity occurred in any of these procedures.

The surgical procedures are listed in Table 2. One patient had video-assisted thoracoscopy (VATS) operation but in all other cases thoracotomy was performed.

\section{Antifungal treatment}

Most patients (12, 55\%) used antifungal medication prior to surgery. In clinical practice, medication schemes were diverse. The preoperative-medication period ranged from 3 weeks to 39 months. Most patients received treatment postoperatively $(19,86 \%)$, the suggested length of the medication varied from 1 to 6 months and in cases of recurrent infection or suspected CCPA or ABPA (allergic bronchopulmonary aspergillosis) continued until death or was considered long term or permanent. Some patients, however, continued the medication for an indefinite time period even after surgery, with the medication stopped only when considerable side-effects occurred. Voriconazole was the most commonly used antifungal medication, but some patients received itraconazole or posaconazole instead. Four patients (18\%) received i.v. treatment peri- or postoperatively with either amphotericin-B or caspofungin. Three patients (14\%) received no antifungal treatment preor postoperatively, because surgery was considered sufficient therapy.

\section{Complications}

No perioperative mortality occurred, and no mortality 


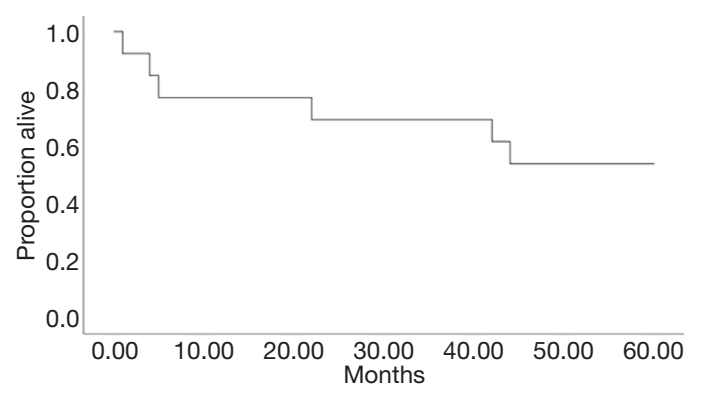

Figure 4 Five-year survival.

within 30 days post-operatively. One in-hospital death, however, occurred; on day 42 one patient died from postoperative complications-he had prolonged air leak and pneumonia and had remained in ICU since surgery. Other postoperative complications included prolonged air leak for over 7 days in five patients (23\%), pneumothorax occurring after hospital discharge in two (9\%) and pneumomediastinum in one (5\%), and postoperative pneumonia in five $(23 \%)$ patients. No postoperative pleuritis or Aspergillus empyema occurred.

\section{Recurrent disease}

Recurrent Aspergillus infection has been confirmed in four patients (18\%) after their surgery. One of them underwent surgery as a palliative procedure because he had recurrent Aspergillus disease (after initial aspergilloma operation in 2001). He was evidently suffering from CCPA and died from Aspergillus infection 6 months after palliative surgery. The three other patients in whom recurrent Aspergillus disease occurred were all asthma patients with elevated Aspergillus-IgE-levels and all were suffering from ABPA and possibly CCPA; some overlapping and transition in these diseases occur $(17,18)$.

None of the three asthma patients who had history of ABPA were on continuous corticosteroid treatment during or shortly prior to the operation. The indication for surgery was haemoptysis on one of these patients, one had been treated for three years with antifungals but she developed bronchopleural fistulae and was operated to control the infection. The third one of these patients also had history of recent renal carcinoma and possibility of pulmonary metastasis supported the decision to operate.

In all these three patients a radiologically evident new aspergilloma has formed in a pulmonary cavity after the initial operation.

\section{Survival}

During the subsequent follow-up until October 2018, of the 22 patients 10 (45\%) died. During the first year after surgery three patients (14\%) died: one of postoperative complications, one of oesophageal carcinoma that he already had at the time of surgery, and one of Aspergillus infection six months after palliative surgery.

All the other $19(86 \%)$ patients were alive 20 months after surgery. The last of the operations evaluated took place in February 2017. Five-year survival could be evaluated in 13 of the 22. Six out of 13 (46\%) died within five years of the surgery including the 3 deaths occurring within one year, 5-year survival is presented in Figure 4. The reasons for death of the 10 patients who died, included pulmonary fibrosis associated with rheumatoid arthritis, lung cancer (3 patients), and GVHD after Hodgkin disease and allogenic stem cell transplantation. In two cases the reason of death could not be concluded from the medical records. In Table 3 are presented the patient characteristics and reasons for death. Only two deaths appear to be related to Aspergillus-disease.

\section{Discussion}

In our post-operative cohort, the recurrence rate of Aspergillus-infection was low. Interestingly, all the recurrent infections in this small group appeared in asthmatics who suffered from ABPA or CCPA and ABPA. All these three patients had cavitary disease with progression although the history of asthma, elevated total IgE and Aspergillus IgElevels suggested the diagnosis of ABPA.

Short-term results of surgery were good, all but one patient surviving the immediate postoperative period. Only one other patient died of recurrent pulmonary aspergillosis, but the indication for his surgery was palliation instead of cure. Other postoperative deaths cannot be considered the result of Aspergillus disease.

Most surgical patients had normal or nearly normal preoperative lung function. Careful selection of patients for surgery has probably resulted in their good overall outcome and few recurrent infections.

The wide variety of surgical procedures ranged from pneumonectomy to palliative fenestration. Surgical procedures were time-consuming, and the postoperative hospital stay was often prolonged. Within four weeks patients recovered well, however, and most of them were cured. The number of complications was reasonable, and no 
Table 3 Patient characteristics, in chronological order of surgery 2004-2017

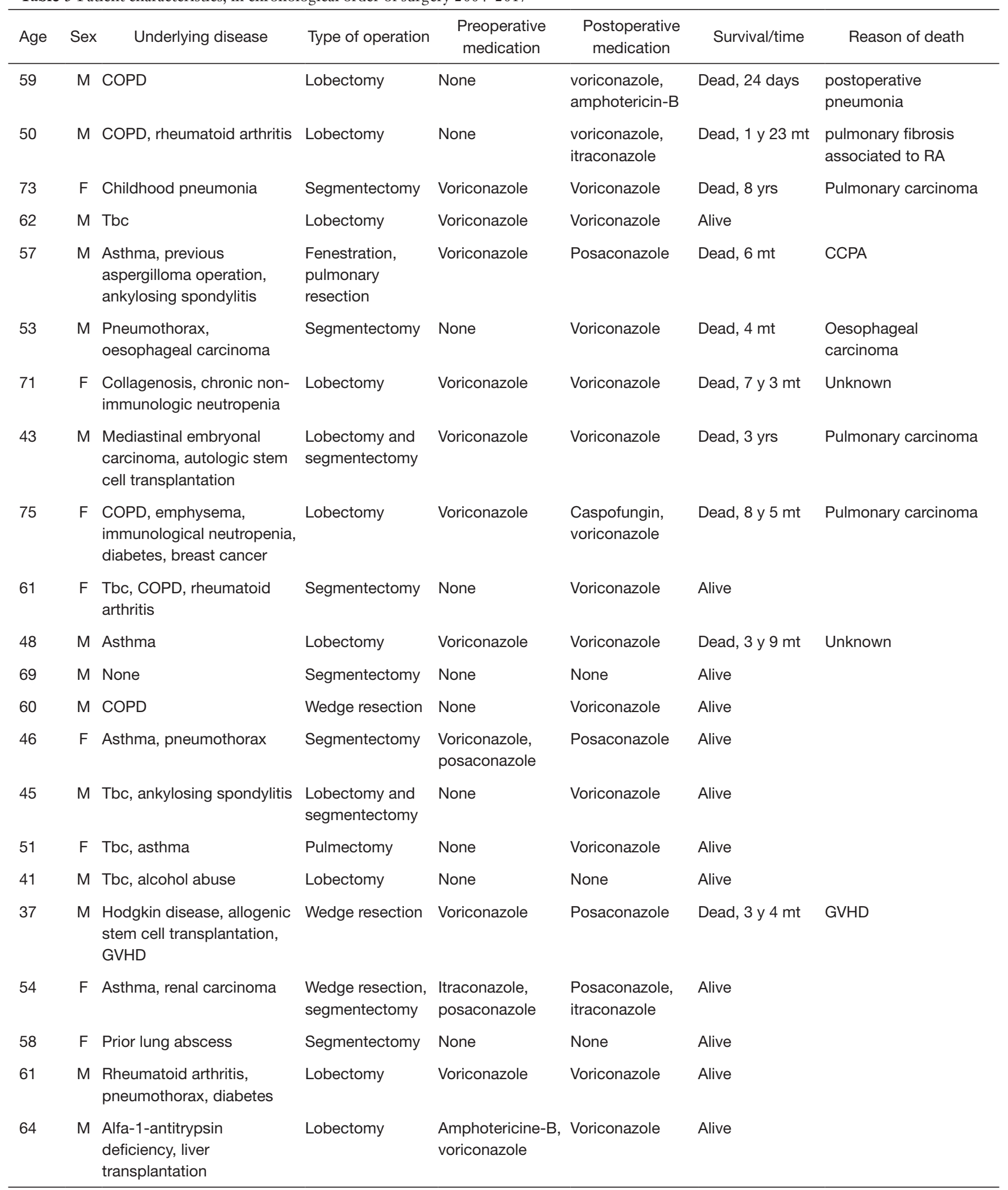

COPD, chronic obstructive pulmonary disease; tbc, tuberculosis; RA, rheumatoid arthritis; GVHD, graft-versus-host disease. 
Aspergillus pleuritis or empyema occurred.

Therapy with antifungals was diverse, and recommendations for medical therapy are clearly needed. Considering medical treatment, our cohort was too small for drawing conclusions regarding the efficacy of antifungal treatment or its length, but our study does provide valuable knowledge of current Finnish practice.

The current IDSA (Infectious Diseases society of America) guidelines, updated in 2016, recommend regarding CCPA: "surgical resection is an option for some patients with localized disease, unresponsive to medical therapy, including those with pan-azole-resistant Aspergillus fumigatusinfection or persistent bemoptysis despite bronchial artery embolization" and state: "the outcomes from surgery are less favorable than those with single aspergilloma and careful risk assessment prior to surgical intervention is required" (19).

Indications for surgery in this study appear to be very similar to those in other studies haemoptysis (45\%) being the commonest indication, as for Farid et al. (8) in Manchester, UK, during 1996-2011, and Muniappan et al. (20) in Massachusetts, USA, during 1980-2010. The proportion for incidental diagnosis of an aspergilloma in these earlier studies was considerably higher than for ours, being $23 \%$ and $37 \%$. Almost all of our patients had preoperatively confirmed aspergilloma diagnosis or strong suspicion of it; only one case of ours being considered incidental. During the observation time 2004-2017 in our study the indications to operate have remained unchanged.

Recurrent infections of Aspergillus in all of these studies were uncommon. For Farid et al. the recurrence rate of Aspergillus disease was 26\% (eight patients), 75\% of whom had CCPA preoperatively, Muniappan et al. reported only four cases $(7 \%)$ of recurrent infection and in our study recurrence rate was $18 \%$. Farid et al. reported four deaths (13\%) for any cause within five years; Muniappan et al. reported five-year survival of $79 \%$. Survival in our study was $54 \%(7 / 13)$ in five years, and of 6 deaths 4 were unrelated to Aspergillus.

In the IDSA guidelines for aspergillosis antifungal therapy is not routinely recommended when surgery is for simple aspergillomas, but they recommend that it be started pre- or perioperatively and continued postoperatively when the risk of surgical spillage of aspergilloma during surgery is moderate (19). The European Respiratory Society/ European Society of Clinical Microbiology and Infectious Diseases (ERS/ESCMID) clinical guidelines for chronic aspergillosis state that "when simple aspergilloma can be resected without spillage of fungal material adjuvant antifungal therapy is not required, but if spillage is anticipated, antifungal therapy can be administered weeks prior to the operation. No evidence, however, exists to support role of antifungal therapy and no recommendations thus can be made, and the use of antifungal therapy should be individualised" (3).

This study suffers from several limitations. Our number of patients is low, since the only operations performed were those with a reasonable risk of surgical complications. According to the literature, surgical treatment for Aspergillus disease is rare. It is understandable that in the case of non-localized disease-as most CCPA is - resection of one site of infection does not lead to ideal results. Patients suffering of progressive disease and CCPA in our hospital have undergone surgery only exceptionally, and many other studies have had a higher number of CCPA patients $(6,8,20,21)$.

The only absolute indication for surgery in aspergilloma is life-threatening massive haemoptysis. Since the aspergilloma is often present in a grossly destroyed lung the possibility of a difficult surgical procedure and complications always has to be a major consideration. An aspergilloma in a pulmonary cavity is a risk for haemoptysis and possibly for more invasive Aspergillus disease $(11,22,23)$. The size of the aspergilloma or clinical picture of the disease does not predict whether life-threatening haemoptysis may occur $(4,24)$. Currently, medical treatment of chronic Aspergillus disease only rarely leads to cure and risk for development of azole resistance may be increased when an aspergilloma exists (25). Moreover, medications are not very well tolerated in long-term use (26). Results of aspergilloma operations appear to be excellent for patients with localized disease, and for those whose expected risk for serious complications in surgery is low or relatively low. Good results occur for surgeries on appropriately selected asymptomatic patients $(7,22,27)$. This was the case also in our study. These patients can often be cured, and no alternative curative treatment exists; if left untreated, these chronic infections lead to high mortality and morbidity, often also despite attempts to treat.

More controversial is the question whether patients with non-localized disease can be helped by surgery. Will surgery help to limit the disease, reduce the burden of the infectious agent, or make it easier to control the disease with antifungals? In CCPA, disease progression may lead to emergencies with massive haemoptysis requiring emergency operations that cannot always be avoided despite the high risk from such operations. The question of timingconsidering high operative risks, and on the other hand, 
progressive disease means high risk of an emergencysometimes in clinical practice leads to difficult decisions with no simple or ideal choices.

Antifungal medication and its duration in surgery for simple aspergillomas still remain questions. Based on the low number and various schemes for antifungal medications regarding our cohort we can neither propose any recommendations on antifungal treatment. No evidence that prolonged treatment with antifungals could be recommended after a successful aspergilloma operation, seems to exist (28-30).

\section{Conclusions}

Surgical treatment can be life-saving and increase quality of life in patients, suffering from haemoptysis. Also, surgery should be seriously considered in those whose disease appears to be restricted and their pulmonary reserve to be well preserved.

\section{Acknowledgments}

This work was supported by the Foundation of the Finnish Anti-Tuberculosis Association, Tampere Tuberculosis Foundation, Nummela Sanatorium Foundation, Väinö and Laina Kivi Foundation and Research Foundation for Pulmonary Diseases (Heli).

\section{Footnote}

Conflicts of Interest: The authors have no conflicts of interest to declare.

Ethical Statement: The authors are accountable for all aspects of the work in ensuring that questions related to the accuracy and integrity of any part of the work are appropriately investigated and resolved. The study protocol was approved by the research board of the Heart and Lung Centre of Helsinki University Hospital (ID HUS/46/2018).

\section{References}

1. Kosmidis C, Denning DW. The clinical spectrum of pulmonary aspergillosis. Thorax 2015;70:270-7.

2. Belcher JR, Plummer NS. Surgery in broncho-pulmonary aspergillosis. Br J Dis Chest 1960;54:335-41.

3. Denning DW, Cadranel J, Beigelman-Aubry C, et al. Chronic pulmonary aspergillosis: Rationale and clinical guidelines for diagnosis and management. Eur Respir J 2016;47:45-68.

4. Jewkes J, Kay PH, Paneth M, et al. Pulmonary aspergilloma: analysis of prognosis in relation to hemoptysis and survey of treatment. Thorax 1983;38:572-8.

5. Camara B, Reymond E, Saint-Raymond C, et al. Characteristics and outcomes of chronic pulmonary aspergillosis: A retrospective analysis of a tertiary hospital registry. Clin Respir J 2015;9:65-73.

6. El Hammoumi MM, Slaoui O, El Oueriachi F, et al. Lung resection in pulmonary aspergilloma: experience of a Moroccan center. BMC Surg 2015;15:114.

7. Akbari JG, Varma PK, Neema PK, et al. Clinical profile and surgical outcome for pulmonary aspergilloma: A single center experience. Ann Thorac Surg 2005;80:1067-72.

8. Farid S, Mohamed S, Devbhandari M, et al. Results of surgery for chronic pulmonary Aspergillosis, optimal antifungal therapy and proposed high risk factors for recurrence - a National Centre's experience. J Cardiothorac Surg 2013;8:180.

9. Faulkner SL, Vernon R, Brown PP, et al. Hemoptysis and Pulmonary Aspergilloma: Operative versus Nonoperative Treatment. Ann Thorac Surg 1978;25:389-92.

10. Kauffman CA. Quandary about treatment of aspergillomas persists. Lancet. 1996;347:1640.

11. Lee JK, Lee YJ, Park SS, et al. Clinical course and prognostic factors of pulmonary aspergilloma. Respirology 2014;19:1066-72.

12. Campbell JH, Winter JH, Richardson MD, et al. Treatment of pulmonary aspergilloma with itraconazole. Thorax 1991;46:839-41.

13. Hammerman KJ, Christianson CS, Huntington I, et al. Spontaneous lysis of aspergillomata. Chest 1973;64:679-9.

14. Aspergilloma and residual tuberculous cavities--the results of a resurvey. Tubercle 1970;51:227-45.

15. Walsh TJ, Anaissie EJ, Denning DW, et al. Treatment of aspergillosis: Clinical practice guidelines of Infectious Diseases Society of America. Clin Infect Dis 2008;46:327-60.

16. Denning DW, Riniotis K, Dobrashian R, et al. Chronic Cavitary and Fibrosing Pulmonary and Pleural Aspergillosis: Case Series, Proposed Nomenclature Change, and Review. Clin Infect Dis 2003;37:S265-80.

17. Lowes D, Chishimba L, Greaves M, et al. Development of chronic pulmonary aspergillosis in adult asthmatics with ABPA. Respir Med 2015;109:1509-15.

18. Smith NL, Denning DW. Underlying conditions in chronic pulmonary aspergillosis including simple aspergilloma. Eur Respir J 2011;37:865-72. 
19. Patterson TF, Thompson GR 3rd, Denning DW, et al. Practice Guidelines for the Diagnosis and Management of Aspergillosis: 2016 Update by the Infectious Diseases Society of America. Clin Infect Dis 2016;63:e1-60.

20. Muniappan A, Tapias LF, Butala P, et al. Surgical therapy of pulmonary aspergillomas: A 30-year north American experience. Ann Thorac Surg 2014;97:432-8.

21. Komori K, Hattori A, Matsunaga T, et al. Feasibility of surgery for pulmonary aspergilloma: analysis of the operative modes. Gen Thorac Cardiovasc Surg 2018;66:276-83.

22. Lejay A, Falcoz PE, Santelmo N, et al. Surgery for aspergilloma: time trend towards improved results? Interact Cardiovasc Thorac Surg 2011;13:392-5.

23. Rafferty P, Biggs BA, Crompton GK, et al. What happens to patients with pulmonary aspergilloma? Analysis of 23 cases. Thorax 1983;38:579-83.

24. Passera E, Rizzi A, Robustellini M, et al. Pulmonary aspergilloma. Clinical aspects and surgical treatment outcome. Thorac Surg Clin 2012;22:345-61.

Cite this article as: Pihlajamaa K, Anttila VJ, Räsänen JV, Kauppi JT, Hodgson U. The fate of aspergilloma patients after surgical treatment-experience from 22 cases. J Thorac Dis 2019;11(10):4298-4307. doi: 10.21037/jtd.2019.09.48
25. Howard SJ, Cerar D, Anderson MJ, et al. Frequency and evolution of azole resistance in Aspergillus fumigatus associated with treatment failure. Emerg Infect Dis 2009; 15:1068-76.

26. Bongomin F, Harris C, Hayes G, et al. Twelve-month clinical outcomes of 206 patients with chronic pulmonary aspergillosis. PLoS One 2018;13:e0193732.

27. Lee JG, Lee CY, Park IK, et al. Pulmonary aspergilloma: Analysis of prognosis in relation to symptoms and treatment. J Thorac Cardiovasc Surg 2009;138:820-5.

28. Zheng S, Li X, Hu B, et al. Is adjuvant antifungal therapy after video-assisted thoracic surgery for pulmonary aspergilloma necessary? J Thorac Dis 2018;10:6060-5.

29. Sagan D, Goździuk K. Surgery for Pulmonary Aspergilloma in Immunocompetent Patients: No Benefit From Adjuvant Antifungal Pharmacotherapy. Ann Thorac Surg 2010;89:1603-10.

30. Benhamed L, Woelffle D. Adjuvant antifungal therapy after pulmonary surgery for aspergilloma: Is it useful? Interact Cardiovasc Thorac Surg 2014;18:835-7. 